\title{
openheart Resting heart rate and incident venous thromboembolism: the Multi-Ethnic Study of Atherosclerosis
}

\author{
Josephine Awotoye, ${ }^{1}$ Oluwaseun E Fashanu, ${ }^{2,3}$ Pamela L Lutsey, ${ }^{4}$ Di Zhao, ${ }^{5}$ \\ Wesley T O'Neal, ${ }^{6}$ Erin D Michos (1) ${ }^{3,5}$
}

\begin{abstract}
- Additional material is published online only. To view please visit the journal online (http://dx.doi.org/10.1136/ openhrt-2019-001080).
\end{abstract}

To cite: Awotoye J, Fashanu OE, Lutsey PL, et al. Resting heart rate and incident venous thromboembolism: the MultiEthnic Study of Atherosclerosis. Open Heart 2020;7:e001080. doi:10.1136/

openhrt-2019-001080

$\mathrm{JA}$ and $\mathrm{OEF}$ contributed equally.

Received 2 May 2019

Revised 7 January 2020

Accepted 27 January 2020

\section{ABSTRACT}

Objective Venous thromboembolism (VTE) is associated with significant morbidity and mortality. Resting heart rate (RHR), which may be modifiable through lifestyle changes, has been shown to be associated with cardiovascular disease risk and with inflammatory markers that have been predictive of VTE incidence.

Methods We examined whether RHR is also associated with VTE incidence independent of these risk factors. We studied 6479 Multi-Ethnic Study of Atherosclerosis participants free from clinical VTE at baseline who had baseline RHR ascertained by 12-lead ECG. VTE events were recorded from hospital records and death certificates using International Classification of Diseases (ICD)-9 and ICD-10 codes. We categorised RHR as $<60,60-69,70-79$ and $\geq 80 \mathrm{bpm}$. We used Cox hazard models to determine the association of incident VTE by RHR.

Results Participants had mean (SD) age of 62 (10) years and RHR of 63 (10) bpm. RHR was cross-sectionally correlated with multiple inflammatory and coagulation factors. There were 236 VTE cases after a median followup of 14 years. Compared with those with $\mathrm{RHR}<60 \mathrm{bpm}$, the $\mathrm{HR}(95 \% \mathrm{Cl})$ for incident VTE for $\mathrm{RHR} \geq 80 \mathrm{bpm}$ was 2.08 (1.31 to 3.30), after adjusting for demographics, physical activity, smoking, diabetes and use of atrioventricular (AV)nodal blockers, aspirin and anticoagulants, and remained significant after further adjustment for inflammatory markers (2.05 (1.29 to 3.26)). Results were similar after excluding those taking AV-nodal blocker medications. There was no effect modification of these associations by sex or age.

Conclusion Elevated RHR was positively associated with VTE incidence after a median of 14 years; this association was independent of several traditional VTE and inflammatory markers.

\section{INTRODUCTION}

Venous thromboembolism (VTE) may present as a deep venous thrombosis (DVT) and/or a pulmonary embolism $(\mathrm{PE})^{1}$ and is associated with significant morbidity and mortality. ${ }^{23}$ Although both diseases (PE and DVT) have different clinical manifestations, they represent the same disease process and share similar risk factors. ${ }^{45}$ The identification of modifiable risk factors is paramount

\section{Key questions}

What is already known about this subject?

- An elevated resting heart rate (RHR) may reflect decreased parasympathetic tone and increased sympathetic stimulation and is associated with increased cardiovascular disease (CVD) risk. Elevated RHR has been associated with inflammatory markers, and alteration in the sympathetic nervous system may predispose to thrombosis. We examined whether RHR was associated with increased incidence of venous thromboembolism (VTE).

What does this study add?

- In a large diverse community cohort of individuals free of VTE, CVD, atrial fibrillation and active cancer at baseline, we demonstrated that RHR was significantly associated with a twofold increased risk of incidence of VTE over a median of 14 years. This association was independent of several demographics, lifestyle and inflammatory factors. RHR also had a significant and positive correlation, though weak, with several inflammatory and coagulation factors cross-sectionally.

How might this impact on clinical practice?

- RHR can readily be measured at clinic visits or through personal fitness trackers; however, it remains underused for the purpose of cardiovascular and thrombosis risk assessments. Furthermore, RHR may be modifiable with improvement in fitness or with pharmacotherapy. Further studies are needed to determine whether the associations of RHR and VTE are causal or secondary to another underlying process, and whether modification of RHR can reduce VTE risk.

to reducing the disease incidence and prevalence. ${ }^{1}$ Resting heart rate (RHR) is an easily assessable clinical variable that is potentially modifiable with lifestyle changes and/or pharmacotherapy. In the previous studies, a higher RHR has been shown to be a significant predictor of all-cause mortality and cardiovascular disease (CVD), independent of traditional risk factors. ${ }^{6-10}$ The association between RHR and CVD also differed by sex. ${ }^{10}$ 
Compared with women, men had a lower average RHR but a greater CVD risk associated with elevated RHR. ${ }^{10}$ However, whether RHR is associated with VTE, specifically, is unknown. RHR has also been shown to be associated with several inflammatory markers, ${ }^{7}{ }^{11}$ which in turn have been predictive of VTE incidence. ${ }^{12} 13$ A prior study also showed a positive and significant correlation between RHR and several coagulation factors. ${ }^{14}$ Taken together, a higher RHR may predispose to a hypercoagulative state, which may lead to a VTE event. However, to the best of our knowledge, at the time we initiated this work, no epidemiological study had yet to evaluate the association between RHR and VTE incidence in a general community-based cohort. Therefore, the aim of our study is to determine the prospective association between RHR and incidence of VTE among participants of the MultiEthnic Study of Atherosclerosis (MESA), who were free of clinical CVD and VTE at baseline and whether this association differs by sex.

\section{METHODS}

The MESA population consists of 6814 men and women who, at recruitment in 2000-2002, were aged 45-84 years and without clinical atherosclerotic CVD, heart failure or atrial fibrillation, and were not undergoing active treatment for cancer. ${ }^{15}$ Participants were of four races/ethnicities including white, black, Hispanic and Chinese, and recruited from six communities in the USA including Los Angeles County, California; Chicago, Illinois; Baltimore/Baltimore County, Maryland; Saint Paul, Minnesota; Forsyth County, North California and Northern Manhattan/Bronx, New York. After baseline (20002002), participants attended up to five additional clinic visits at exam 2 (2002-2004), exam 3 (2004-2005), exam 4 (2005-2007), exam 5 (2010-2012) and exam 6 (20162018). Additionally, they took part in regular phone calls which queried recent hospitalisations. The design of the MESA study has been previously published. ${ }^{15}$

After exclusions for missing baseline RHR $(n=48)$, missing covariates in our primary model $(n=258)$ or missing VTE follow-up data $(n=29)$, a total of 6479 participants were included for our primary analysis for incident VTE (online supplementary figure 1). In a supplemental analysis conducted to help establish potential mechanisms, we also examined the cross-sectional association of RHR with several plasma haemostatic factors and endothelial markers (further described below in this section). Some of these biomarkers were measured in the entire cohort, while others were measured in a random sample of a 1000 MESA participants. ${ }^{16}$ The baseline characteristics of these 'MESA 1000' participants did not differ from that of the entire MESA cohort.

For the primary analysis, we used data regarding RHR and clinical and laboratory covariates that were obtained at the MESA baseline examination. Participants were instructed to fast for at least 12 hours and avoid heavy exercise before the exam. A 12-lead ECG obtained at rest was used to record the RHR of participants at baseline.

Surveys and questionnaires were used to gather information on age, sex, race/ethnicity, education and smoking status. Information on physical activity was obtained by asking questions on time and frequency of physical activity during the week using a survey instrument adapted from the Cross-Cultural Activity Participation Study, ${ }^{17}$ and total minutes of moderate and/or vigorous exercise per week were calculated. Body mass index (BMI) was measured as the weight (kilograms)/ height $\left(\mathrm{kg} / \mathrm{m}^{2}\right)$. Diabetes was based on self-report, medication history as well as a fasting glucose level of $\geq 126 \mathrm{mg}$ / dL. A medication inventory approach ${ }^{18}$ was used to determine atrioventricular (AV)-nodal blocker use (defined as participants who were on either beta-blockers, verapamil or diltiazem), aspirin and oral anticoagulants, as well as use of antihypertensive and lipid-lowering therapies. The Chronic Kidney Disease Epidemiology equation was used to calculate the estimated glomerular filtration rate (eGFR). ${ }^{19}$

Serum concentration of high-sensitivity $\mathrm{C}$ reactive protein (hsCRP) and fibrinogen were measured using the Dade Behring Nephelometer II Analyzer System (BNII) (Deerfield, Illinois, USA). ${ }^{11}$ Interleukin-6 (IL-6) serum concentration was measured using ultrasensitive ELISA (Quantikine HS Human IL-6 Immunoassay, R\&D Systems). ${ }^{11}$ D-dimer was measured using Sta-R analyzer (Liatest D-DI; Diagnostica Stago, Parsippany, New Jersey, USA). ${ }^{20}$

As previously described, plasma haemostatic factors and endothelial markers were measured in various samples of the MESA cohort. ${ }^{16}$ At baseline, in the entire cohort, factor VIII and plasmin-antiplasmin (PAP) were measured, whereas plasminogen activator inhibitor-1 (PAI-1), von Willebrand factor (VWF), soluble thrombomodulin (STM) and E-selectin were measured only in the 'MESA 1000' random sample. ${ }^{16}$ Intercellular adhesion molecule 1 (ICAM-1) was measured from the 'MESA 1000' sample and also from all participants enrolled before February 2003. ${ }^{16}$ Due to missing data and exclusions, the numbers included slightly varied across these biomarkers.

Participants were followed from study baseline (20002002) to 2015. They were contacted by telephone every 9-12 months to obtain information regarding selfreported hospitalisation, and the medical records from these hospitalisations were obtained for review. Incident VTE events were identified via these hospitalisation records and also from death certificates by using International Classification of Diseases (ICD)-9 and ICD-10 codes. These codes were predetermined by a panel of VTE experts in the MESA coordinating centre. They were chosen to be consistent with the ICD codes used for the Longitudinal Investigation of Thromboembolism (LITE) study $^{21}$ whose goal was to determine the incidence of VTEs in two community-based cohorts: the Atherosclerosis Risk in Communities (ARIC) ${ }^{22}$ and the Cardiovascular Health 
Study cohorts ${ }^{23}$ (see online supplementary table 1 for the specific ICD-9 and ICD-10 codes used).

We stratified baseline characteristics by the presence/absence of incident VTE. We present continuous variables as mean $\pm \mathrm{SD}$ or median (25-75th percentile) for normally distributed and skewed variables, respectively. We present categorical variables as frequency (percentage). We used two samples t-test, Mann-Whitney test or $\mathrm{X}^{2}$ test to describe differences between groups as appropriate. We used multivariable-adjusted Cox proportional hazards regression models to determine the HRs and $95 \%$ CIs of incident VTE by RHR groups categorised by previously established cut points $(<60,60-69$,
$70-79$ and $\geq 80 \mathrm{bpm}) .{ }^{102425}$ We tested for trends across RHR categories by using an ordinal variable for each RHR category and modelling this as a continuous variable for association with VTE. We also examined RHR as a continuous variable per $10 \mathrm{bpm}$ increment. We tested non-violation of the Cox proportional assumption using Schoenfeld residuals.

We used progressively adjusted models. In model 1, we adjusted for demographic variables of age (continuous), sex (men; women) and race/ ethnicity (white; black; Hispanic; Chinese). Model 2 (our primary analytical model) was further adjusted for socioeconomic and lifestyle factors such as education (<high school; high

Table 1 Baseline characteristics of study participants by incident venous thromboembolism (VTE): the Multi-Ethnic Study of Atherosclerosis, 2000-2002*

\begin{tabular}{|c|c|c|c|c|}
\hline Baseline characteristics & Total & No VTE & VTE & $P$ value \\
\hline N & 6479 & 6243 & 236 & - \\
\hline Heart rate, bpm & $63.1 \pm 9.6$ & $63 \pm 9.6$ & $64.6 \pm 11.1$ & 0.01 \\
\hline Heart rate, bpm* & $62(56-69)$ & $62(56-69)$ & $63(56-73)$ & 0.04 \\
\hline Age, years & $62.1 \pm 10.2$ & $62 \pm 10.2$ & $66.6 \pm 9.4$ & $<0.001$ \\
\hline Women & $3423(52.8 \%)$ & $3296(52.8 \%)$ & $127(53.8 \%)$ & 0.76 \\
\hline Race/ethnicity & & & & $<0.001$ \\
\hline White & $2504(38.7 \%)$ & $2403(38.5 \%)$ & $101(42.8 \%)$ & - \\
\hline Black & $1757(27.1 \%)$ & $1668(26.7 \%)$ & $89(37.7 \%)$ & - \\
\hline Hispanic & $1431(22.1 \%)$ & $1394(22.3 \%)$ & $37(15.7 \%)$ & - \\
\hline Chinese & $787(12.2 \%)$ & $778(12.5 \%)$ & $9(3.8 \%)$ & - \\
\hline Education & & & & 0.69 \\
\hline Less than high school & $1176(18.2 \%)$ & $1134(18.2 \%)$ & $42(17.8 \%)$ & - \\
\hline High school or vocational school & $2683(41.4 \%)$ & $2579(41.3 \%)$ & $104(44.1 \%)$ & - \\
\hline College, graduate or professional school & $2620(40.4 \%)$ & $2530(40.5 \%)$ & $90(38.1 \%)$ & - \\
\hline Smoking status & & & & 0.33 \\
\hline Never & $3268(50.4 \%)$ & $3160(50.6 \%)$ & $108(45.8 \%)$ & - \\
\hline Former & $2381(36.8 \%)$ & $2285(36.6 \%)$ & $96(40.7 \%)$ & - \\
\hline Current & $830(12.8 \%)$ & $798(12.8 \%)$ & $32(13.6 \%)$ & - \\
\hline $\mathrm{BMI}, \mathrm{kg} / \mathrm{m}^{2}$ & $28.3 \pm 5.4$ & $28.2 \pm 5.4$ & $30.5 \pm 6.2$ & $<0.001$ \\
\hline Physical activity, MET-minutes/week† & $4020(1980-7515)$ & $4035(1980-7545)$ & $3554(1864-6544)$ & 0.13 \\
\hline Diabetes & $814(12.6 \%)$ & $777(12.5 \%)$ & $37(15.7 \%)$ & 0.14 \\
\hline eGFR, $\mathrm{ml} / \mathrm{min}$ per $1.73 \mathrm{~m}^{2}$ & $77.7 \pm 16.2$ & $77.9 \pm 16.1$ & $73.5 \pm 18.4$ & $<0.001$ \\
\hline AV nodal blocker medication & $829(12.8 \%)$ & $792(12.7 \%)$ & $37(15.7 \%)$ & 0.18 \\
\hline Anticoagulant use & $24(0.4 \%)$ & $20(0.3 \%)$ & $4(1.7 \%)$ & 0.001 \\
\hline Aspirin use & $1631(25.2 \%)$ & $1556(24.9 \%)$ & $75(31.8 \%)$ & 0.02 \\
\hline hsCRP, mg/L† & $1.9(0.8-4.2)$ & $1.9(0.8-4.2)$ & $2.5(1.2-4.5)$ & 0.002 \\
\hline Interleukin-6, pg/mL† & $1.2(0.8-1.9)$ & $1.2(0.8-1.9)$ & $1.4(0.9-2.6)$ & $<0.001$ \\
\hline Fibrinogen, $\mathrm{mg} / \mathrm{dL} \dagger$ & 337 (295-388) & 337 (294-387) & 346 (309-407) & 0.01 \\
\hline D-dimer, $\mu \mathrm{g} / \mathrm{mL} \dagger$ & $0.2(0.1-0.4)$ & $0.2(0.1-0.4)$ & $0.4(0.2-0.6)$ & $<0.001$ \\
\hline
\end{tabular}

*Data are presented as mean \pm SD for continuous variables and as frequency (percentage) for categorical variables, unless otherwise specified. $\mathrm{P}$ values derived from two samples t-test or Mann-Whitney test for continuous variables and $\mathrm{X}^{2}$ test for categorical variables. †Data presented as median (25-75th percentile).

$\mathrm{AV}$, atrioventricular; BMI, body mass index; bpm, beats per minute; eGFR, estimated glomerular filtration rate; hsCRP, high-sensitivity $\mathrm{C}$ reactive protein; MET, metabolic equivalent of task. 
Table 2 Risk of incident venous thromboembolism (VTE) associated with resting heart rate: the Multi-Ethnic Study of Atherosclerosis, 2000-2015

\begin{tabular}{|c|c|c|c|c|c|c|}
\hline Resting heart rate & $\leq 60 \mathrm{bpm}$ & 60-69 bpm & 70-79 bpm & $\geq 80 \mathrm{bpm}$ & P for trend & $\begin{array}{l}\text { Per } 10 \text { bpm } \\
\text { increment }\end{array}$ \\
\hline $\mathrm{N}$ & 2434 & 2540 & 1143 & 362 & - & 6479 \\
\hline VTE; n (\%) & $83(3.4)$ & $75(3.0)$ & $52(4.6)$ & $26(7.2)$ & - & $236(3.6)$ \\
\hline Person-years & 30559 & 31820 & 14013 & 4219 & - & 80610 \\
\hline Incidence rate $(95 \% \mathrm{Cl})^{*}$ & 2.72 (2.19 to 3.37 ) & 2.36 (1.88 to 2.96$)$ & 3.71 (2.83 to 4.87 ) & 6.16 (4.20 to 9.05$)$ & - & 2.93 (2.58 to 3.33 ) \\
\hline \multicolumn{7}{|l|}{ HRs $(95 \% \mathrm{Cl}) \dagger$} \\
\hline Model 1 & 1 (reference) & 0.92 (0.67 to 1.25$)$ & 1.47 (1.04 to 2.08 ) & 2.49 (1.60 to 3.88$)$ & $<0.001$ & $1.24(1.09$ to 1.41$)$ \\
\hline Model 2 & 1 (reference) & 0.88 (0.65 to 1.21$)$ & $1.31(0.92$ to 1.87$)$ & 2.01 (1.28 to 3.15$)$ & 0.004 & $1.17(1.02$ to 1.33$)$ \\
\hline Model 3 & 1 (reference) & 0.88 (0.64 to 1.20$)$ & 1.33 (0.93 to 1.91) & 2.08 (1.31 to 3.30$)$ & 0.004 & 1.18 (1.03 to 1.35$)$ \\
\hline Model 4 & 1 (reference) & 0.90 (0.66 to 1.24) & 1.37 (0.95 to 1.96$)$ & 2.05 (1.29 to 3.26$)$ & 0.003 & $1.18(1.03$ to 1.35$)$ \\
\hline
\end{tabular}

Bolded values are statistically significant, $\mathrm{p}<0.05$.

*Incidence rate is unadjusted and per 1000 person-years.

†Adjusted HRs per models as follows:

Model 1 adjusted for age, sex and race/ ethnicity.

Model 2: model 1 plus education, BMI and log (physical activity).

Model 3: model 2 plus smoking status, diabetes, eGFR, AV nodal blocker use, anticoagulant use and aspirin use.

Model 4: model 3 plus log (hsCRP), log (IL-6), log (fibrinogen) and log (D-dimer).

AV, atrioventricular; BMI, body mass index; bpm, beats per minute; eGFR, estimated glomerular filtration rate; hsCRP, high-sensitivity C reactive protein; IL-6, interleukin-6; MET, metabolic equivalent of task.

school or vocational school; college, graduate or professional school), BMI (continuous) and physical activity level (metabolic equivalent of task (MET) minutes /week of moderate or vigorous activity; log-transformed continuous). Model 3 was additionally adjusted for cigarette smoking (current; former; never), diabetes (yes; no), eGFR (continuous), use of AV nodal blocking agents (yes; no), aspirin (yes; no) and anticoagulants (yes; no).
Note that the use of oral anticoagulants was very low in MESA ( $0.4 \%$ of our analytic sample), as MESA excluded those with clinical CVD and atrial fibrillation at baseline. We did not adjust for participants with oral contraceptive or hormone therapy use as data were only limited to women and was not associated with VTE in this cohort (not shown). In a final model, model 4, we further adjusted for the inflammatory and coagulation markers

Table 3 Risk of incident venous thromboembolism associated with resting heart rate excluding participants on AV-nodal blockers: the Multi-Ethnic Study of Atherosclerosis), 2000-2015

\begin{tabular}{|c|c|c|c|c|c|c|}
\hline Resting heart rate & $\leq 60 \mathrm{bpm}$ & 60-69 bpm & 70-79 bpm & $\geq 80$ bpm & P for trend & $\begin{array}{l}\text { Per } 10 \text { bpm } \\
\text { increment }\end{array}$ \\
\hline $\mathrm{N}$ & 2011 & 2252 & 1055 & 332 & - & 5650 \\
\hline Person-years & 25499 & 28414 & 12937 & 3909 & - & 70760 \\
\hline Incidence rate $(95 \% \mathrm{Cl})^{*}$ & 2.43 (1.90 to 3.12$)$ & 2.22 (1.73 to 2.84 ) & 3.86 (2.93 to 5.10$)$ & 6.14 (4.11 to 9.16$)$ & - & 2.81 (2.45 to 3.23$)$ \\
\hline Model 2 & 1 (reference) & 0.90 (0.63 to 1.28$)$ & 1.48 (1.01 to 2.16$)$ & 2.01 (1.24 to 3.27 ) & 0.002 & $1.22(1.06$ to 1.41$)$ \\
\hline Model 3 & 1 (reference) & 0.92 (0.64 to 1.31) & 1.51 (1.03 to 2.22$)$ & 2.14 (1.31 to 3.49$)$ & 0.001 & 1.24 (1.07 to 1.44$)$ \\
\hline Model 4 & 1 (reference) & 0.95 (0.67 to 1.36$)$ & 1.56 (1.06 to 2.31$)$ & 2.13 (1.30 to 3.48$)$ & 0.001 & 1.25 (1.08 to 1.45$)$ \\
\hline
\end{tabular}

Bolded values are statistically significant, $\mathrm{P}<0.05$

*Incidence rate is unadjusted and per 1000 person-years.

†Adjusted HRs per Models as follows:

Model 1 is adjusted for age, sex and race/ ethnicity.

Model 2: model 1 plus education, BMl and log (physical activity).

Model 3: model 2 plus smoking status, diabetes, eGFR, AV nodal blocker use, anticoagulant use and aspirin use.

Model 4: model 3 plus log (hsCRP), log (IL-6), log (fibrinogen) and log (D-dimer).

$\mathrm{AV}$, atrioventricular; BMI, body mass index; bpm, beats per minute; eGFR, estimated glomerular filtration rate; hsCRP, high-sensitivity C reactive protein; IL-6, interleukin-6; MESA, Multi-Ethnic Study of Atherosclerosis; MET, metabolic equivalent of task; VTE, venous thromboembolism. 


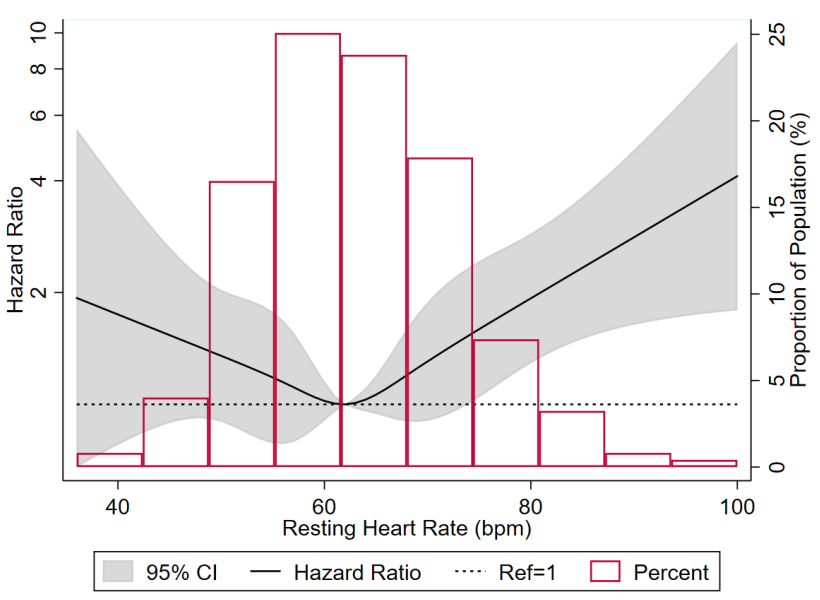

Figure 1 Restricted cubic spline* showing association of resting heart rate with incident venous thromboembolism in MESA, 2000-2015 *High extreme values of RHR (>100 bpm) were excluded $(n=4)$ from graphical display. The histogram shows the distribution (proportion) of participants by RHR. The restricted cubic spline shows the HRs $(95 \% \mathrm{Cl})$ for the risk of incident VTE associated with RHR (continuous). The spline was generated using a Cox proportional hazards model, with median RHR (62 bpm) as reference, and with knots at 5th, 27.5th, 50th, 72.5th and 95th percentiles. The dotted line represents an HRof 1 (ref). The model was adjusted for age (continuous), sex (male;female), race/ ethnicity (white; black; Hispanic; Chinese), education (<high school; high school or vocational school; college, graduate or professional school), BMI (continuous) and physical activity level (metabolic equivalent-minutes /week of moderate or vigorous activity; log-transformed continuous). MESA, MultiEthnic Study of Atherosclerosis; MET, metabolic equivalent of task; RHR, resting heart rate; VTE, venous thromboembolism.

(all log-transformed, continuous) of hsCRP, IL-6, fibrinogen and D-dimer, which may play an intermediary role in the relationship between RHR and VTE.

To further characterise the relationship between RHR and incident VTE, we used restricted cubic splines centred at the median RHR (62 bpm) with five knots placed at the 5th, 27.5th, 50th, 72.5th and 95th percentiles adjusted for variables in our main analytic model (model 2).

We performed several sensitivity analyses. First, we explored if the association between RHR and VTE differed by sex based on a priori hypotheses. ${ }^{10}$ We also examined interactions by age groups (per 10-year increments). Second, we excluded participants on AV-nodal blocker medications. Third, we modelled RHR and some covariates in our models as time-varying, with variables updated at MESA exams 2, 3, 4 and/or 5, as available (of note, since VTE events were recorded through 2015, data from MESA exam 6 which began in 2016 was not included). Lastly, to evaluate for potential mechanisms, we examined the partial correlations of RHR with various inflammatory and coagulation factors in MESA, using Spearman's correlation (r), after adjusting for age, sex, and race/ethnicity. ${ }^{16}$ We considered a $\mathrm{p}<0.05$ to be statistically significant, and performed our analyses using Stata V.15.

\section{RESULTS}

The mean (SD) age of participants $(\mathrm{n}=6479)$ was $62(10)$ years and RHR of 63 (10) bpm; 53\% were women, 39\% white, $27 \%$ black, 22\% Hispanic and 12\% Chinese Americans. Participants who had VTE at follow-up had a significantly higher mean RHR, age, BMI and lower eGFR at baseline when compared with participants who did not develop a VTE. Participants who developed incident VTE also had higher median hsCRP, IL-6, fibrinogen and D-dimer levels and a higher proportion were using anticoagulants and aspirin at baseline. Sex, educational status, smoking status, physical activity, diabetes and use of AV-nodal medications at baseline were not statistically significantly related to incident VTE (table 1 ). The baseline characteristics according to RHR groups are presented in online supplementary table 2. Participants with a higher RHR were more likely to be women, have lower average physical activity, have a higher prevalence of diabetes and have higher levels of the inflammatory markers.

After a median of 14 years of follow-up, there were 236 incident cases of VTE. Of these cases, one event was a VTE mortality. The unadjusted VTE incidence rate $(95 \% \mathrm{CI})$ in this population who were free from VTE at baseline was 2.93 (2.58 to 3.33) per 1000 person-years. The HRs (95\% CIs) for incident VTE after accounting for baseline demographics (model 1) comparing the participants with RHR of 60-69 bpm, 70-79 bpm and $\geq 80 \mathrm{bpm}$ to participants with RHR $\leq 60 \mathrm{bpm}$ was 0.92 (0.67 to 1.25 ), 1.47 (1.04 to 2.08) and 2.49 (1.60 to 3.88), respectively, ( $\mathrm{p}$ for trend $<0.001$ ). Compared with $\mathrm{RHR} \leq 60 \mathrm{bpm}$, the association between elevated RHR $\geq 80 \mathrm{bpm}$ with VTE incidence remained statistically significant in our main model (model 2) which further accounted for socioeconomic and lifestyle factors (HR $2.01(1.28,3.15))$ and did not differ significantly by sex ( $\mathrm{p}$ for interaction $=0.10$ ) or by age groups ( $\mathrm{p}$ for interaction $=0.64$, online supplementary table 3 ). In our fully adjusted model (model 4) which further accounted for inflammatory markers associated with VTE, the HR (95\% CI) for incident VTE was 2.05 (1.29 to 3.26) for RHR $\geq 80 \mathrm{bpm}$ compared with $\leq 60 \mathrm{bpm}$. Results also remained statistically significant when we modelled RHR as a continuous variable in all models (table 2).

Sensitivity analysis among only the participants who were not taking AV-nodal blocker medications at baseline showed a higher risk of incident VTE for RHR 70-79 $\mathrm{bpm}$ and $\geq 80 \mathrm{bpm}$ versus RHR $\leq 60 \mathrm{bpm}$, compared with primary analysis, with HRs of 1.56 (1.06 to 2.31) and 2.13 (1.30 to 3.48 ), respectively, in our fully adjusted model (model 4, table 3 ).

The mean RHR was also noted to be consistently higher in participants who developed VTE across the MESA follow-up visits compared with those who did not develop 
Table 4 Partial correlations of resting heart rate with inflammatory/coagulation factors: the Multi-Ethnic Study of Atherosclerosis, 2000-2002*

\begin{tabular}{|c|c|c|c|c|c|c|}
\hline \multirow[b]{2}{*}{ Marker } & \multicolumn{2}{|c|}{ Whole cohort } & \multicolumn{2}{|c|}{ Males } & \multicolumn{2}{|c|}{ Females } \\
\hline & $\mathbf{N}$ & $r$ ( $p$ value) & $\mathbf{N}$ & $r$ (p value) & $\mathbf{N}$ & $r$ ( $p$ value) \\
\hline hs-CRP & 6714 & $0.15(p<0.001)$ & 3173 & $0.18(p<0.001)$ & 3541 & $0.12(p<0.001)$ \\
\hline Interleukin-6 & 6576 & $0.15(p<0.001)$ & 3101 & $0.17(p<0.001)$ & 3475 & $0.13(p<0.001)$ \\
\hline Fibrinogen & 6721 & $0.15(p<0.001)$ & 3181 & $0.19(p<0.001)$ & 3540 & $0.13(p<0.001)$ \\
\hline D-dimer & 6721 & $0.03(p=0.005)$ & 3182 & $0.04(p=0.02)$ & 3539 & $0.03(p=0.12)$ \\
\hline VWF & 992 & $0.10(p=0.002)$ & 425 & $0.07(p=0.14)$ & 567 & $0.12(p=0.01)$ \\
\hline Factor VIII & 6717 & $0.12(p<0.001)$ & 3179 & $0.11(p<0.001)$ & 3538 & $0.12(p<0.001)$ \\
\hline PAP & 6584 & $0.05(p<0.001)$ & 3119 & $0.08(p<0.001)$ & 3465 & $0.002(p=0.91)$ \\
\hline PAl-1 & 968 & $0.13(p<0.001)$ & 418 & $0.16(p=0.002)$ & 550 & $0.11(p=0.01)$ \\
\hline STM & 992 & $0.03(p=0.39)$ & 426 & $-0.01(p=0.77)$ & 566 & $0.08(p=0.07)$ \\
\hline E-selectin & 994 & $0.16(p<0.001)$ & 426 & $0.21(p<0.001)$ & 568 & $0.11(p=0.01)$ \\
\hline ICAM-1 & 2610 & $0.07(p<0.001)$ & 1146 & $0.07(p=0.03)$ & 1464 & $0.07(p=0.01)$ \\
\hline
\end{tabular}

r, Spearman's correlation.

${ }^{*}$ Adjusted for age, sex (except in sex stratified analysis), and race/ethnicity.

hsCRP, high sensitivity C reactive protein; ICAM-1, Intercellular adhesion molecule 1; PAI-1, plasminogen activator inhibitor-1; PAP, plasminantiplasmin; STM, soluble thrombomodulin; VWF, von Willebrand factor.

VTE (online supplementary table 4). However, there was no significant change in mean heart rate across time in participants who developed VTE $(\mathrm{p}=0.14)$ as compared with the trend seen among those who did not develop VTE $(p<0.001)$. In sensitivity analyses, when RHR was modelled as a time-varying covariate updated at each of the MESA follow-up visits, results were similar to our primary models (online supplementary table 5).

We also visually depicted the association using a restricted cubic spline model (figure 1), adjusted for model 2 covariates. The association appeared to be U-shaped, with decreased VTE risk for RHR less than the median value of $62 \mathrm{bpm}$ (although with wide CIs) but increased VTE risk associated with elevated RHR when RHR $>$ median.

Among participants who had data for RHR and inflammatory/coagulation factors (table 4), we found RHR to be significantly and positively associated with a number of inflammatory/coagulation factors after adjusting for age, sex and race/ethnicity, including hsCRP, IL-6, fibrinogen, D-dimer, VWF, Factor VIII, PAP, PAI-1, E-selectin and ICAM-1. RHR was not significantly associated with STM.

\section{DISCUSSION}

In a large diverse community cohort of individuals free of VTE, CVD, atrial fibrillation or active cancer at baseline, we demonstrated that RHR was significantly associated with the incidence of VTE independent of several demographics, lifestyle and inflammatory factors. There was no interaction by sex or age, and the association appeared stronger after excluding participants on AV-nodal blocker medications. We also demonstrated that RHR had a significant and positive correlation, though weak, with several inflammatory and coagulation factors cross-sectionally including hsCRP, IL-6, fibrinogen, D-dimer, VWF, FVIII, PAP, PAI-1, E-selectin and ICAM-1.

RHR is a vital sign that is routinely measured at most clinical encounters, as well as self-monitored by individuals using fitness trackers and mobile devices. Prior studies have demonstrated that elevated RHR is associated with an increased risk of CVD, all-cause mortality ${ }^{6-10}$ valvular heart disease ${ }^{2526}$ and cognitive decline. ${ }^{24}$ However, there is little literature available on its role with VTE.

There are several plausible biological mechanisms by which an elevated RHR may contribute to VTE. Prior studies have shown that elevated RHR is positively associated with concentrations of inflammatory and coagulation markers, ${ }^{711} 13$ as our present study also demonstrates, and this may provide some explanation as to the role of elevated RHR in predicting VTE risk. In a study by Whelton $e t a l$, which included participants enrolled in the MESA study, it was shown that elevated RHR was associated with higher levels of hs-CRP, IL- 6 and fibrinogen. ${ }^{11}$ In another study in the Danish population, Jensen et al demonstrated that RHR was associated with hsCRP and fibrinogen. ${ }^{7}$ RHR may also be associated with activation of haemostatic and thrombotic factors. In a pilot study of participants with mitral stenosis and atrial fibrillation, participants with higher heart rates $(>100 \mathrm{bpm})$ were found to have significantly higher levels of coagulation factors (prothrombin fragment $1+2$, thrombin antithrombin III and PAI) when compared with participants with lower heart rates $(\leq 100 \mathrm{bpm}) .{ }^{14}$

RHR is thought to reflect a balance of sympathetic and parasympathetic nervous systems. The parasympathetic system predominates in resting states, so an elevated RHR may be reflective of decreased parasympathetic tone and increased sympathetic stimulation. Alteration in the sympathetic nervous system may predispose to thrombosis 
risk, ${ }^{27} 28$ which may be another mechanism linking RHR to VTE formation. At rest, individuals with increased physical fitness tend to have higher parasympathetic tone and better autonomic function, which contributes to a lower RHR. ${ }^{911}$ However, a prior study found that there remained an association of RHR with mortality risk even after adjusting for measured fitness (METS on treadmill testing). ${ }^{10}$ RHR has also been associated with progression of valvular calcification and stenosis, which may be due to mechanical shear stress from enhanced cardiac output. ${ }^{25} 26$

In sum, our findings re-enforce the association of RHR with inflammatory and coagulation processes. In addition, we now newly show an association of RHR with future VTE risk, independent of several traditional VTE and inflammatory risk factors. This suggests that the risk of RHR with VTE may not fully be explained by its association with inflammatory markers and may be due to other causes. However, it is important to note that the inflammatory markers assessed in this study were measured only once at baseline, and adjustment for baseline values may not capture the long-term exposure of these markers or their intraindividual variability. More work is needed to be done to establish these mechanisms. However, to confirm that the associations we found were not spurious, after completing the above analysis in MESA, we then sought out to confirm whether RHR was associated with VTE in another large prospective cohort, the ARIC study, which notably had adjudicated VTE outcomes. This confirmatory work from our group was recently published and similar associations were found for RHR and incident VTE, with HRs (95\% CI) of 1.44 (1.01 to 2.06) comparing RHR of $\geq 80 \mathrm{bpm}$ to $<60 \mathrm{bpm}$ and 1.11 (1.02 to 1.21) per 10 bpm increment in RHR. ${ }^{29}$

Our study has many strengths including the prospective design and utilisation of data from the well-characterised MESA cohort, which allowed us to rigorously adjust for numerous potentially confounding demographic, lifestyle and VTE risk factors. We studied a large sample of racially/ethnically diverse men and women over a prolonged time course of 14 years to study the long-term associations between baseline RHR (and time-varying RHR) and incident VTE. Nevertheless, our findings have some limitations, which are worth noting. First, cases of VTE were identified from medical records using ICD codes and were not adjudicated. This may have led to some false positives or negatives. However, as noted above, our confirmatory follow-up analysis in the ARIC cohort, which did have validated VTE outcomes, found similar results. ${ }^{29}$ Second, we made use of RHR at baseline for our primary analysis, which may not be representation of RHR during follow-up. Nevertheless, our sensitivity analysis using RHR at multiple follow-up visits still showed significant associations between elevated RHR and VTE risk. Third, the observational design does limit causal inference. Although we attempted to adjust for multiple VTE risk factors, residual confounding may still exist due to the multifactorial nature and complex pathophysiology of VTE. For example, elevated RHR may be a manifestation for someone in a poorer health state.

\section{CONCLUSIONS}

In summary, in a large multiethnic community-based cohort, we have demonstrated that elevated RHR was associated with an increased risk of incident VTE and with an increased level of several inflammatory and coagulation factors. Mechanisms for this association may be activation of inflammatory pathway, sympathetic nervous system and mechanical shear stress. However, further studies are needed to determine whether the associations of RHR and VTE are causal or secondary to another underlying process. RHR, which is an easily assessed clinical variable, is underused for vascular risk prediction. Whether the modification of RHR by lifestyle or pharmacological intervention would reduce the future risk of VTE is unknown and warrants further exploration.

\section{Author affiliations}

${ }^{1}$ Department of Biology, University of Minnesota, Minneapolis, Minnesota, USA

${ }^{2}$ Department of Medicine, Saint Agnes Hospital, Baltimore, Maryland, USA

${ }^{3}$ The Ciccarone Center for the Prevention of Cardiovascular Disease, Johns Hopkins School of Medicine, Baltimore, Maryland, USA

${ }^{4}$ Division of Epidemiology and Community Health, School of Public Health, University of Minnesota, Minneapolis, Minnesota, USA

${ }^{5}$ Department of Epidemiology, Johns Hopkins Bloomberg School of Public Health, Baltimore, Maryland, USA

${ }^{6}$ Department of Medicine, Division of Cardiology, Emory University School of Medicine, Atlanta, Georgia, USA

Acknowledgements The authors thank the staff and participants of MESA study for their important contributions. A full list of participating MESA investigators and institutions can be found at http://www.mesa-nhlbi.org.

Contributors The concept of this research study was designed by JA, OEF and EDM. OEF performed the statistical analyses under the guidance of DZ. JA and EDM wrote the initial draft. OEF, PLL, DZ and WTO contributed significant intellectual content. All authors approved of the final draft of the manuscript.

Funding EDM and DZ were supported by the Blumenthal Scholars Fund for Preventive Cardiology Research at Johns Hopkins University. This MESA study was supported by contracts HHSN268201500003I, N01-HC-95159, N01-HC-95160, N01-HC-95161, N01-HC-95162, N01-HC-95163, N01-HC-95164, N01-HC-95165, N01-HC-95166, N01-HC-95167, N01-HC-95168 and N01-HC-95169 from the National Heart, Lung and Blood Institute (NHLBI), and by grants UL1-TR-000040, UL1-TR-001079 and UL1-TR-001420 from NCATS.

\section{Competing interests None declared.}

Patient consent for publication The MESA study was approved by the Institutional Review Boards at each study site, and participants provided written informed consent.

Ethics approval Johns Hopkins School of Medicine Institutional Review Board (IRB) Multi-Ethnic Study of Atherosclerosis NA_00030361/CR00010390.

Provenance and peer review Not commissioned; externally peer reviewed.

Data availability statement Data may be obtained from a third party and are not publicly available. The MESA study participates in data sharing through the National Heart, Lung, Blood Institute (NHLBI) Biologic Specimen and Data Repository Coordinating Center (BioLINCC). Requests for access to the data can be made through their website: https://biolincc.nhlbi.nih.gov/studies/mesa/.

Open access This is an open access article distributed in accordance with the Creative Commons Attribution 4.0 Unported (CC BY 4.0) license, which permits others to copy, redistribute, remix, transform and build upon this work for any purpose, provided the original work is properly cited, a link to the licence is given, and indication of whether changes were made. See: https://creativecommons.org/ licenses/by/4.0/. 
ORCID iD

Erin D Michos http://orcid.org/0000-0002-5547-5084

\section{REFERENCES}

1 Heit JA, Spencer FA, White RH. The epidemiology of venous thromboembolism. J Thromb Thrombolysis 2016;41:3-14.

2 Beckman MG, Hooper WC, Critchley SE, et al. Venous thromboembolism: a public health concern. Am J Prev Med 2010;38:S495-501.

3 Benjamin EJ, Virani SS, Callaway CW, et al. Heart disease and stroke Statistics-2018 update: a report from the American heart association. Circulation 2018;137:e67-492.

4 Wolberg AS, Rosendaal FR, Weitz JI, et al. Venous thrombosis. Nat Rev Dis Primers 2015;1:15006.

5 Lutsey PL, Norby FL, Alonso A, et al. Atrial fibrillation and venous thromboembolism: evidence of bidirectionality in the Atherosclerosis risk in Communities study. J Thromb Haemost 2018;16:670-9.

6 Jensen MT, Suadicani P, Hein $\mathrm{HO}$, et al. Elevated resting heart rate, physical fitness and all-cause mortality: a 16-year follow-up in the Copenhagen male study. Heart 2013;99:882-7.

7 Jensen MT, Marott JL, Allin KH, et al. Resting heart rate is associated with cardiovascular and all-cause mortality after adjusting for inflammatory markers: the Copenhagen City heart study. Eur J Prev Cardiol 2012;19:102-8.

8 Zhang D, Shen X, Qi X. Resting heart rate and all-cause and cardiovascular mortality in the general population: a meta-analysis. Can Med Assoc J 2016;188:E53-63.

9 Aladin Al, Al Rifai M, Rasool SH, et al. The association of resting heart rate and incident hypertension: the Henry Ford Hospital exercise testing (fit) project. Am J Hypertens 2016;29:251-7.

10 Aladin Al, Whelton SP, Al-Mallah $\mathrm{MH}$, et al. Relation of resting heart rate to risk for all-cause mortality by gender after considering exercise capacity (the Henry Ford exercise testing project). Am J Cardiol 2014;114:1701-6.

11 Whelton SP, Narla V, Blaha MJ, et al. Association between resting heart rate and inflammatory biomarkers (high-sensitivity C-reactive protein, interleukin-6, and fibrinogen) (from the multi-ethnic study of atherosclerosis). Am J Cardiol 2014;113:644-9.

12 Folsom AR, Lutsey PL, Astor BC, et al. C-reactive protein and venous thromboembolism. A prospective investigation in the ARIC cohort. Thromb Haemost 2009;102:615-9.

13 Saghazadeh A, Rezaei N. Inflammation as a cause of venous thromboembolism. Crit Rev Oncol Hematol 2016;99:272-85.

14 Yusuf J, Goyal M, Mukhopadhyay S, et al. Effect of heart rate control on coagulation status in patients of rheumatic mitral stenosis with atrial fibrillation - a pilot study. Indian Heart J 2015;67:S40-5.
15 Bild DEet al. Multi-Ethnic study of atherosclerosis: objectives and design. Am J Epidemiol 2002;156:871-81.

16 Lutsey PL, Cushman M, Steffen LM, et al. Plasma hemostatic factors and endothelial markers in four racial/ethnic groups: the MESA study. J Thromb Haemost 2006;4:2629-35.

17 Ainsworth BE, Irwin ML, Addy CL, et al. Moderate physical activity patterns of minority women: the cross-cultural activity participation study. J Womens Health Gend Based Med 1999;8:805-13.

18 Smith NL, Psaty BM, Heckbert SR, et al. The reliability of medication inventory methods compared to serum levels of cardiovascular drugs in the elderly. J Clin Epidemiol 1999;52:143-6.

19 Levey AS, Bosch JP, Lewis JB, et al. A more accurate method to estimate glomerular filtration rate from serum creatinine: a new prediction equation. modification of diet in renal disease Study Group. Ann Intern Med 1999;130:461-70.

20 Folsom AR, Delaney JAC, Lutsey PL, et al. Associations of factor VIII, D-dimer, and plasmin-antiplasmin with incident cardiovascular disease and all-cause mortality. Am J Hematol 2009;84:349-53

21 Cushman M, Tsai AW, White $\mathrm{RH}$, et al. Deep vein thrombosis and pulmonary embolism in two cohorts: the longitudinal investigation of thromboembolism etiology. Am J Med 2004;117:19-25.

22 Anon. The Atherosclerosis risk in communities (ARIC) study: design and objectives. The ARIC Investigators. Am J Epidemiol 1989;129:687-702

23 Fried LP, Borhani NO, Enright P, et al. The cardiovascular health study: design and rationale. Ann Epidemiol 1991;1:263-76.

24 Wang S, Fashanu OE, Zhao D, et al. Relation of Elevated Resting Heart Rate in Mid-Life to Cognitive Decline Over 20 Years (from the Atherosclerosis Risk in Communities [ARIC] Study). Am J Cardiol 2019;123:334-40.

25 Amoakwa K, Fashanu OE, Tibuakuu M, et al. Resting heart rate and the incidence and progression of valvular calcium: the multi-ethnic study of atherosclerosis (MESA). Atherosclerosis 2018;273:45-52.

26 de Oliveira Moraes AB, Stähli BE, Arsenault BJ, et al. Resting heart rate as a predictor of aortic valve stenosis progression. Int J Cardiol 2016;204:149-51.

27 Pöyhönen-Alho M, Joutsi-Korhonen L, Lassila R, et al. Alterations of sympathetic nervous system, coagulation and platelet function in gestational diabetes. Blood Coagul Fibrinolysis 2012;23:508-13.

28 Preckel D, von Känel R. Regulation of hemostasis by the sympathetic nervous system: any contribution to coronary artery disease? Heart Drug 2004;4:123-30.

29 Folsom AR, Lutsey PL, Pope ZC, et al. Resting heart rate and incidence of venous thromboembolism. Res Pract Thromb Haemost 2019;27. 